\title{
No association between body composition and cognition in ambulatory persons with multiple sclerosis: A brief report
}

\author{
Brian M. Sandroff, MS; Elizabeth A. Hubbard, MS; Lara A. Pilutti, PhD; Robert W. Motl, PhD* \\ Department of Kinesiology and Community Health, University of Illinois at Urbana-Champaign, Urbana, IL
}

\begin{abstract}
There is evidence that body fat is inversely associated with cognitive functioning in adults from the general population, and this has been associated with systemic inflammation. The association between body fat and cognition might further be augmented in the presence of an immune-mediated, inflammatory disease such as multiple sclerosis (MS). This cross-sectional study investigated the associations between objective measures of body composition and cognitive function in 60 persons with MS. Participants underwent a neurological examination for generating Expanded Disability Status Scale scores, followed by the Brief International Cognitive Assessment in Multiple Sclerosis neuropsychological battery for measurement of cognitive processing speed, verbal learning and memory, and visual learning and memory. Whole-body fat mass, percent body fat, lean body mass, and bone mineral density were measured using dualenergy X-ray absorptiometry. Whole-body fat mass and percent body fat were not associated with any cognitive outcome (all $p>$ 0.41 ). However, lean body mass was associated with cognitive processing speed $(p<0.03)$, and bone mineral density was associated with cognitive processing speed and verbal learning and memory. Those associations were attenuated and nonsignificant after controlling for age and Expanded Disability Status Scale scores $(p>0.13)$. Body composition might not represent a target of interventions for improving cognitive processing speed or learning and memory in MS.
\end{abstract}

Key words: body composition, body fat, cognition, cognitive processing speed, disability, DXA, learning and memory, multiple sclerosis, neuropsychological, obesity.

\section{INTRODUCTION}

There has been recent interest in the association between measures of body fat and cognitive function [1]. This is based on reports of statistically significant associations between obesity and cognitive function in animal work and studies involving adults from the general population [13]. For example, obese rats tend to perform worse on learning and memory tasks than nonobese rats, and this has been associated with systemic inflammation [1,4]. Two recent reviews describe impairments in executive function and learning and memory in obese adults from the general population based on body mass index (BMI) [1-2], and this too might be associated with systemic inflammation. Another

Abbreviations: BICAMS $=$ Brief International Cognitive Assessment in Multiple Sclerosis, BMD = bone mineral density, BMI = body mass index, BVMT-R = Brief Visuospatial Memory Test-Revised, CNS = central nervous system, CVLT-2 = California Verbal Learning Test-2, DMT = disease-modifying treatment, DXA = dual-energy $\mathrm{X}$-ray absorptiometry, EDSS = Expanded Disability Status Scale, MS = multiple sclerosis, SD = standard deviation, SDMT = Symbol-Digit Modalities Test.

*Address all correspondence to Robert W. Motl, PhD; University of Illinois at Urbana-Champaign, Department of Kinesiology and Community Health, 233 Freer Hall, 906 S Goodwin Ave, Urbana, IL 61801; 217-265-0886; fax: 217244-7322. Email: robmotl@illinois.edu

http://dx.doi.org/10.1682/JRRD.2014.09.0208 
review describes that such systemic inflammation might further lead to central inflammation in the brain [3]. That review describes the possibility of central inflammation resulting in synaptic remodeling and neurodegeneration within the brain, and those processes might present as cognitive dysfunction [3]. The association between increasing body fat and worsening cognitive function might further be augmented in the presence of an immune-mediated, inflammatory neurological disorder. However, the association between body fat measures and cognition has not been wellstudied in neurological populations, for example, multiple sclerosis (MS).

MS is a common neurological disease of the central nervous system (CNS) that is initially characterized by episodic areas of inflammation that results in demyelination and eventual transection of axons [5]. The CNS damage manifests as the accumulation of physical and cognitive disability [6]. There is evidence that persons with MS have similar obesity rates and body composition as the general population (i.e., high prevalence of overweight/obesity) [7-9]. Cognitive dysfunction too is a major consequence of MS, with upwards of 50 percent of patients demonstrating cognitive impairment based on neuropsychological testing [10].

Despite having comparable body composition characteristics to adults of the general population, perhaps having poor body composition is particularly burdensome for cognitive functioning in adults with MS based on the inflammatory nature of the disease. Indeed, one study reports that higher bone mineral density (BMD) of the femur was associated with better visual learning and memory in 56 ambulatory persons with MS, although that study does not report outcomes of body fat [11]. That study provides preliminary support for an association between body composition outcomes and cognitive function in MS. However, there have been no studies directly examining the association between body fat outcomes and cognitive performance in persons with MS. Such an examination might be important for identifying potential targets for improving cognition in this population, given that there has not been overwhelming support for pharmacological or nonpharmacological approaches for managing cognitive impairment in MS [10,12-13].

To that end, the current cross-sectional study investigated the associations between measures of body composition (based on dual-energy X-ray absorptiometry [DXA]) and domains of cognitive function in ambulatory persons with MS.

\section{METHODS}

\section{Participants}

The current study represents a secondary analysis of data (i.e., investigation of nonprimary outcomes) from a single cross-sectional study examining fitness outcomes in ambulatory persons with MS. ${ }^{*}$ The final sample included 60 persons with MS, and we have described the recruitment, enrollment, and inclusion criteria in detail elsewhere [14]. Of note, all participants provided physician's approval for enrollment into the study. All participants further had a diagnosis of MS that was physician-verified and were between the ages of 18 and $64 \mathrm{yr}$, ambulatory with or without assistance, and relapse free during the $30 \mathrm{~d}$ period before enrollment. All participants demonstrated a low risk for contraindications of physical activity based on the Physical Activity Readiness Questionnaire [15].

\section{Primary Measures}

\section{Height and Weight}

Height and weight were measured to the nearest $0.1 \mathrm{~cm}$ or $\mathrm{kg}$, respectively, using a scale-stadiometer unit (Detecto model 3P7044; Webb City, Missouri). Participants wore lightweight clothing suitable for exercise (including shoes) while assessing height and weight. This was expressed as BMI (kilograms per meter squared).

\section{Dual-Energy X-Ray Absorptiometry}

Whole body composition (i.e., fat mass, percent body fat, lean body mass, BMD) was assessed by DXA using a Hologic QDR 4500A bone densitometer (software version 11.2; Bedford, Massachusetts). Accuracy of the densitometer was verified by scanning the manufacturer's hydroxyapatite spine phantom of a known density. All scans were analyzed by the same investigator and verified by a second investigator. Whole-body fat mass and lean body mass (i.e., whole-body mass minus fat mass) are expressed in grams and percent body fat, whereas whole-body BMD is expressed in grams/centimeters.

\footnotetext{
*Pilutti LA, Sandroff BM, Klaren R, Learmonth YC, Platta M, Hubbard EA, Stratton M, Motl RW. Physical fitness across the disability spectrum in multiple sclerosis: A comparison of different testing modalities. J Neurol Phys Ther. 2015. In press.
} 


\section{Cognitive Function}

We administered the Brief International Cognitive Assessment in Multiple Sclerosis (BICAMS) neuropsychological battery as previously described [16]. Tests included in the BICAMS are the Symbol-Digit Modalities Test (SDMT) [17] as a measure of cognitive processing speed, California Verbal Learning Test-2 (CVLT-2) [18] as a measure of verbal learning and memory, and the Brief Visuospatial Memory Test-Revised (BVMT-R) [19] as a measure of visual learning and memory. The primary outcomes from each of those tests are expressed as raw scores.

\section{Disability Status}

A Neurostatus-certified researcher derived Expanded Disability Status Scale (EDSS) [20] scores, based on neurological examination, for describing the clinical characteristics of the sample and possible inclusion as a covariate.

\section{Disease-Modifying Treatment Use}

We included a single self-report item regarding diseasemodifying treatment (DMT) use and have described this measure previously [14]. Briefly, participants indicated yes or no regarding currently using a DMT. If participants indicated current DMT use, there was an additional space for providing the specific medication and dosage.

\section{Procedure}

The study procedure was approved by a university institutional review board, and all participants provided written informed consent. The procedure included a single session for collecting all data. Participants initially underwent the neurological examination for generating EDSS scores, followed by administration of the BICAMS neuropsychological battery. Participants then completed a demographics questionnaire; this was followed by measurement of height and weight by a member of the research team. Participants then underwent a whole-body scan on the densitometer for measurement of body composition outcomes. Participants wore hospital scrubs or lightweight clothing free of metal and removed all jewelry prior to being scanned. All participants received payment upon completion of the testing session.

\section{Data Analysis}

The data were analyzed using SPSS version 21.0 (IBM Corporation; Armonk, New York). Demographic and clinical characteristics are presented in text and tables as mean \pm standard deviation (SD), unless otherwise noted. The primary analyses involved bivariate Spearman rho $\left(r_{s}\right)$ rank-order correlations among measures of body composition and cognition because this minimizes the effect of outliers or nonnormality on bivariate correlations [21]. Correlation coefficients of 0.1, 0.3, and 0.5 were interpreted as small, moderate, and large, respectively [22]. If those correlations were statistically significant, we then performed nonparametric partial correlations $\left(p r_{s}\right)$, controlling for covariates (i.e., age, sex, EDSS scores) based on joint associations with body composition and cognitive outcomes.

\section{RESULTS}

\section{Participant Characteristics}

Demographic and clinical characteristics of the sample are presented in Table 1. Briefly, the sample consisted of mostly women (73.3\%) with relapsing-remitting MS (76.7\%) and moderate disability based on a median EDSS score of 4.5 (range 1.5-6.5). Of the sample, 83.3 percent reported using a DMT (i.e., interferon beta-1a, interferon beta- $1 \mathrm{~b}$, glatiramer acetate, natalizumab). The mean BMI of the sample was $27.2 \pm 6.8 \mathrm{~kg} / \mathrm{m}^{2}$. This indicates that, on average, the sample was overweight based on the BMI cutoffs of 24.9 to $29.9 \mathrm{~kg} / \mathrm{m}^{2}$ [23] and is similar to other larger samples of persons with MS [7-8]. Further, 16 persons with MS (i.e., 26.7\%) were obese based on a BMI of at least $30.0 \mathrm{~kg} / \mathrm{m}^{2}$ [23].

\section{Table 1.}

Demographic and clinical characteristics of 60 persons with multiple sclerosis (MS).

\begin{tabular}{lc}
\hline \multicolumn{1}{c}{ Variable } & Overall MS Sample \\
\hline Age $(\mathrm{yr})$ & $52.5 \pm 7.2$ \\
Sex, $n(\%$ Female) & $44 / 60(73.3)$ \\
BMI $\left(\mathrm{kg} / \mathrm{m}^{2}\right)$ & $27.2 \pm 6.9$ \\
MS Type, $n(\%)$ & \\
$\quad$ Relapsing-Remitting MS & $46 / 60(76.7)$ \\
$\quad$ Progressive MS & $14 / 60(23.3)$ \\
Disease Duration (yr) & $13.2 \pm 8.8$ \\
EDSS, Median (Range) & $4.5(1.5-6.5)$ \\
DMT Use, $n$ (\%) & $50 / 60(83.3)$ \\
\hline Note: All data presented as mean \pm standard deviation unless otherwise noted. \\
BMI = body mass index, DMT = disease-modifying treatment, EDSS = \\
Expanded Disability Status Scale.
\end{tabular}




\section{Body Composition and Cognitive Characteristics}

Body composition (measured by DXA) and cognitive outcomes are presented in Table 2. The current sample had whole-body fat mass, percent body fat, lean body mass, BMD, SDMT, CVLT-2, and BMVT-R values similar to those of other samples of persons with MS [9,24-25]. The distributions of whole-body fat mass and lean body mass were nonnormal based on large, positive skewness values. The current sample demonstrated slowed cognitive processing speed based on SDMT scores approximately 1.16 SD units below the normative score for healthy controls [25]. The current sample further demonstrated poor visual learning and memory based on BVMT-R scores approximately $0.95 \mathrm{SD}$ units below the normative score for healthy controls [25].

\section{Correlations Between Measures of Body Composition and Cognitive Function}

The correlations among whole-body fat mass, percent body fat, lean body mass, BMD, and cognitive function are presented in Table 3. Whole-body fat mass and percent

Table 2.

Descriptive body composition and cognitive characteristics of 60 persons with multiple sclerosis.

\begin{tabular}{lc}
\hline \multicolumn{1}{c}{ Variable } & $\begin{array}{c}\text { Mean } \pm \text { Standard } \\
\text { Deviation }\end{array}$ \\
\hline Whole-Body Fat Mass (g) & $27,077.5 \pm 13,561.9$ \\
Body Fat (\%) & $33.6 \pm 9.8$ \\
Lean Body Mass (g) & $50,519.8 \pm 10,741.7$ \\
BMD (g/cm) & $1.1 \pm 0.1$ \\
SDMT & $50.6 \pm 12.9$ \\
CVLT-2 & $54.6 \pm 12.9$ \\
BVMT-R & $21.4 \pm 7.1$ \\
BMD = whole-body bone mineral density, BVMT-R $=$ Brief Visuospatial \\
Memory Test-Revised, CVLT-2 = California Verbal Learning Test-2, SDMT = \\
Symbol-Digit Modalities Test.
\end{tabular}

body fat were not associated with SDMT $\left(\left|r_{s}\right|<0.11, p>\right.$ 0.41), CVLT-2 $\left(\left|r_{s}\right|<0.05, p>0.73\right)$, or BVMT-R $\left(\left|r_{s}\right|<\right.$ $0.10, p>0.45)$ scores. There further were no statistically significant differences in SDMT $(t(58)=0.82, p=0.42)$, CVLT-2 $(t(58)=0.36, p=0.72)$, or BVMT-R $(t(58)=1.08$, $p=0.29)$ scores between obese and nonobese persons with MS. However, lean body mass was significantly associated with SDMT scores $\left(r_{s}=0.29, p=0.03\right)$ but not CVLT-2 $\left(r_{s}=0.07, p=0.60\right)$ or BVMT-R $\left(r_{s}=0.15, p=0.24\right)$ scores. This association was small to moderate in magnitude, such that higher amounts of lean body mass were associated with faster cognitive processing speed. We then identified EDSS scores (but not age or sex) as a covariate of lean body mass and SDMT scores based on being jointly associated with those outcomes. The association between lean body mass and SDMT scores was attenuated and nonsignificant $\left(p r_{s}=0.14, p=0.30\right)$ after controlling for EDSS scores.

Whole-body BMD was associated with SDMT $\left(r_{s}=\right.$ 0.29, $p=0.03)$ and CVLT-2 $\left(r_{s}=0.31, p=0.02\right)$ scores but not BVMT-R scores $\left(r_{s}=0.10, p=0.44\right)$. Those associations were small to moderate in magnitude, such that higher BMD values were associated with faster cognitive processing speed and better verbal learning and memory. We then identified age and EDSS scores (but not sex) as covariates of whole-body BMD and SDMT scores, and EDSS scores only as a covariate of whole-body BMD and CVLT-2 scores. After controlling for age and EDSS scores, the association between whole-body BMD and SDMT scores was attenuated and nonsignificant $\left(p r_{s}=0.08, p=\right.$ $0.56)$. The association between whole-body BMD and CVLT-2 scores was attenuated and nonsignificant $\left(p r_{\mathrm{s}}=\right.$ $0.20, p=0.13$ ) after controlling for EDSS scores.

Table 3.

Correlations among body composition and cognitive outcomes in 60 persons with multiple sclerosis.

\begin{tabular}{lccccccc}
\hline Variable & Fat Mass & \% Fat & LBM & BMD & SDMT & CVLT-2 & BVMT-R \\
\hline Fat Mass & - & & & & & & \\
\% Fat & $0.92^{*}$ & - & & & & \\
LBM & $0.28^{*}$ & -0.06 & - & & & \\
BMD & 0.09 & -0.01 & $0.31^{*}$ & $-9^{*}$ & - & \\
SDMT & -0.01 & -0.11 & $0.29^{*}$ & $0.29^{*}$ & $0.53^{*}$ & $-\bar{C}^{*}$ & - \\
CVLT-2 & 0.01 & 0.05 & 0.07 & $0.31^{*}$ & $0.30^{*}$ & $0.66^{*}$ \\
BVMT-R & 0.10 & 0.08 & 0.10 & 0.10 &
\end{tabular}

${ }^{*}$ Statistical significance at $p<0.05$.

$\%$ Fat = whole-body fat percentage, BMD = whole-body bone mineral density, BVMT-R = Brief Visuospatial Memory Test-Revised, CVLT-2 = California Verbal Learning Test-2, Fat Mass = whole-body fat mass, LBM = lean body mass, SDMT = Symbol-Digit Modalities Test. 


\section{DISCUSSION}

The current cross-sectional study examined the associations among outcomes of body composition and cognitive function in 60 ambulatory persons with MS. Whole-body fat mass and percent body fat were not associated with any cognitive outcome. Further, measures of cognitive processing speed and learning and memory did not differ as a function of obesity. However, lean body mass and wholebody BMD were associated with cognitive measures (i.e., SDMT and CVLT-2 scores), although those associations were attenuated and nonsignificant after controlling for covariates (i.e., age and EDSS scores). This suggests that poor body composition might not be an explanatory factor for cognitive dysfunction in ambulatory persons with MS despite the immune-mediated, inflammatory nature of the disease. The present results suggest that measures of body composition, particularly fat mass and percent body fat, might not be ideal targets of interventions for managing cognitive function in ambulatory MS.

The current results are not entirely consistent with results from studies of obesity and cognitive function in the general population of adults. There is an emerging but conflicting body of research examining associations between obesity and neuropsychological deficits [2-3]. Indeed, some studies actually report that obesity (i.e., excess body fat) might exert a protective effect against cognitive decline in aging humans $[1,26]$. Such equivocal evidence in the general population suggests that, overall, there might not be a definitive association between measures of body fat and cognition. The present results do not indicate a definitive association between body composition and cognition, independent of age and disability status, in ambulatory persons with MS. Because this line of research is clearly in its infancy, additional research might be necessary for further confirming the potential relationship between body fat and cognition in this population.

There is evidence that multiple domains of physical fitness (i.e., aerobic capacity, muscular strength) are associated with cognitive processing speed in persons with mild-to-moderate MS disability [14,27]. There further is evidence of cognitive-motor coupling in ambulatory MS, such that cognitive function and mobility might be interrelated constructs in this population [28]. This is partially consistent with the current findings of an association between lean body mass and SDMT scores and associations between BMD (i.e., a presumed correlate of mobility in MS [29]) and SDMT and CVLT-2 scores.
However, those associations were attenuated and nonsignificant after controlling for covariates of age and disability status. This was somewhat unexpected since physical fitness (i.e., aerobic and muscular fitness) and mobility outcomes are associated with body composition outcomes across the MS disability spectrum [30] and there is preliminary evidence of associations between measures of BMD and learning and memory in this population [11]. Body composition represents another domain of fitness that has not been well-studied relative to cognitive function in persons with MS. Although poor body composition (i.e., increased body fat, decreased lean body mass, decreased BMD) is associated with comorbid conditions (e.g., cardiovascular disease, type-II diabetes, osteoporosis) [7-9,29] in this population, similar to physiological deconditioning (i.e., reductions in aerobic and muscular fitness) [30], it remains unclear whether or not those outcomes have a direct influence on cognitive functioning in ambulatory persons with MS.

The role of age and disability status relative to the potential relationship of body composition and cognition among ambulatory persons with MS is unclear. In the current study, we identified EDSS scores (i.e., disability status) as covariate of lean body mass and SDMT scores and BMD and CVLT-2 scores, respectively. We identified age and EDSS scores as covariates of BMD and SDMT scores. Although lean body mass was associated with cognitive processing speed, and BMD was associated with cognitive processing speed and verbal learning and memory, those associations became attenuated and nonsignificant after controlling for age and EDSS covariates. This suggests that increasing lean body mass and BMD are not ideal candidates for primary outcomes in interventions for improving cognition in ambulatory persons with MS since age and disability status are seemingly driving those associations. Interestingly, previous studies have described that body composition does not seem to vary as a function of disability status among ambulatory persons with MS [31-32]; however, there is wellestablished evidence that those with worse MS disability generally perform worse on neuropsychological tests [10]. Further, it is well established that increasing age influences both BMD and cognitive performance in general. Future research efforts should carefully consider those age and disability variables when examining potential body composition correlates of cognitive functioning in this population. 
This is the first study examining the association between objective measures of body fat and validated neuropsychological tests of cognitive functions in persons with MS. Nevertheless, there are several limitations to the current study. This study is a secondary analysis of data that were collected for other purposes and permits only a crosssectional analysis of body composition and cognitive outcomes. Given the cross-sectional experimental design (i.e., all data collected in a single testing session), there is the possibility that participants were not familiar with the instructions of the cognitive measures, such that performance might have been downwardly biased. We do not believe this to be a major issue because the SDMT, CVLT-2, and BVMT-R have been validated in persons with MS and were administered with standardized instructions [33]. We did not control for nutritional factors (i.e., caffeine intake, fasting) or measures of fatigue and depression. Those variables may have influenced both body composition and cognitive performance. We did not include a measure of free-living physical activity behavior (i.e., accelerometry) as a potential covariate of body composition and cognition. This limits the strength of the current results since physical activity might be an important factor for both body composition [24] and cognition [34] among ambulatory persons with MS. Further, we did not include a measure of systemic/central inflammation in the current study. Given the potential role of inflammation in the obesity/cognition relationship in the general population, and the importance of DMTs (i.e., powerful anti-inflammatory drugs) in MS, future studies should evaluate this as a possible mechanism for the potential association between body composition and cognition in MS. Another study limitation is that we did not include healthy controls matched by age, sex, height, and weight in order to examine the specificity of the associations between measures of body composition and cognitive function. We note that the results of the current study might not be generalizable to nonambulatory persons with MS because there is evidence of a potentially different pattern of body composition characteristics among wheelchair users with MS [29]. Finally, we did not include a measure of executive function because this domain of cognition is seemingly the most affected in obese adults of the general population [2]. Assessments of this nature should be included in future studies examining the relationships between body composition and cognitive functioning in ambulatory persons with MS.

\section{CONCLUSIONS}

Overall, results from this study indicated that body composition outcomes were not predictive of cognitive function after controlling for age and disability status in persons with MS. Body composition might not represent an ideal outcome for interventions aiming to improve cognitive processing speed and learning and memory in MS.

\section{ACKNOWLEDGMENTS}

\section{Author Contributions:}

Study concept and design: B. M. Sandroff, E. A. Hubbard, L. A. Pilutti, R. W. Motl.

Acquisition of data: B. M. Sandroff, E. A. Hubbard, L. A. Pilutti, R. W. Motl.

Analysis and interpretation of data: B. M. Sandroff, E. A. Hubbard, R. W. Motl.

Drafting of manuscript: B. M. Sandroff, E. A. Hubbard, R. W. Motl. Critical revision of manuscript for important intellectual content:

B. M. Sandroff, E. A. Hubbard, L. A. Pilutti, R. W. Motl.

Obtained funding: L. A. Pilutti, R. W. Motl.

Financial Disclosures: The authors have declared that no competing interests exist.

Funding/Support: This material was based on work supported by the National Multiple Sclerosis Society (grant IL 0003).

Institutional Review: The study procedure was approved by a University Institutional Review Board and all participants provided written informed consent.

Participant Follow-Up: The authors plan to inform participants of the publication of this study.

\section{REFERENCES}

1. Smith E, Hay P, Campbell L, Trollor JN. A review of the association between obesity and cognitive function across the lifespan: Implications for novel approaches to prevention and treatment. Obes Rev. 2011;12(9):740-55. [PMID:21991597]

2. Castanon N, Lasselin J, Capuron L. Neuropsychiatric comorbidity in obesity: Role of inflammatory processes. Front Endocrinol (Lausanne). 2014;5:74. [PMID:24860551] http://dx.doi.org/10.3389/fendo.2014.00074

3. Miller AA, Spencer SJ. Obesity and neuroinflammation: A pathway to cognitive impairment. Brain Behav Immun. 2014;42:10-21. [PMID:24727365] http://dx.doi.org/10.1016/j.bbi.2014.04.001

4. Farr SA, Yamada KA, Butterfield DA, Abdul HM, Xu L, Miller NE, Banks WA, Morley JE. Obesity and hypertriglyceridemia produce cognitive impairment. Endocrinology. 
2008;149(5):2628-36. [PMID:18276751]

http://dx.doi.org/10.1210/en.2007-1722

5. Trapp BD, Nave KA. Multiple sclerosis: An immune or neurodegenerative disorder? Annu Rev Neurosci. 2008;31: 247-69. [PMID:18558855]

http://dx.doi.org/10.1146/annurev.neuro.30.051606.094313

6. Confavreux C, Vukusic S. Natural history of multiple sclerosis: A unifying concept. Brain. 2006;129(Pt 3):606-16. [PMID:16415308] http://dx.doi.org/10.1093/brain/awl007

7. Khurana SR, Bamer AM, Turner AP, Wadhwani RV, Bowen JD, Leipertz SL, Haselkorn JK. The prevalence of overweight and obesity in veterans with multiple sclerosis. Am J Phys Med Rehabil. 2009;88(2):83-91.

[PMID:19169174]

http://dx.doi.org/10.1097/PHM.0b013e318194f8b5

8. Marrie R, Horwitz R, Cutter G, Tyry T, Campagnolo D, Vollmer T. High frequency of adverse health behaviors in multiple sclerosis. Mult Scler. 2009;15(1):105-13.

[PMID:18845651]

http://dx.doi.org/10.1177/1352458508096680

9. Slawta JN, Wilcox AR, McCubbin JA, Nalle DJ, Fox SD, Anderson G. Health behaviors, body composition, and coronary heart disease risk in women with multiple sclerosis. Arch Phys Med Rehabil. 2003;84(12):1823-30.

[PMID:14669190]

http://dx.doi.org/10.1016/S0003-9993(03)00466-0

10. Benedict RH, Zivadinov R. Risk factors for and management of cognitive dysfunction in multiple sclerosis. Nat Rev Neurol. 2011;7(6):332-42. [PMID:21556031] http://dx.doi.org/10.1038/nrneurol.2011.61

11. Batista S, Teter B, Sequeira K, Josyula S, Hoogs M, Ramanathan M, Benedict RH, Weinstock-Guttman B. Cognitive impairment is associated with reduced bone mass in multiple sclerosis. Mult Scler. 2012;18(10):1459-65.

[PMID:22419674] http://dx.doi.org/10.1177/1352458512440206

12. O’Brien AR, Chiaravalloti N, Goverover Y, Deluca J. Evidenced-based cognitive rehabilitation for persons with multiple sclerosis: A review of the literature. Arch Phys Med Rehabil. 2008;89(4):761-69. [PMID:18374010] http://dx.doi.org/10.1016/j.apmr.2007.10.019

13. Amato MP, Langdon D, Montalban X, Benedict RH, DeLuca J, Krupp LB, Thompson AJ, Comi G. Treatment of cognitive impairment in multiple sclerosis: Position paper. J Neurol. 2013;260(6):1452-68. [PMID:23180174] http://dx.doi.org/10.1007/s00415-012-6678-0

14. Sandroff BM, Pilutti LA, Benedict RH, Motl RW. Association between physical fitness and cognitive function in multiple sclerosis: Does disability status matter? Neurorehabil Neural Repair. 2015;29(3):214-23.
[PMID:25009224]

http://dx.doi.org/10.1177/1545968314541331

15. Thomas S, Reading J, Shephard RJ. Revision of the Physical Activity Readiness Questionnaire (PAR-Q). Can J Sport Sci. 1992;17(4):338-45. [PMID:1330274]

16. Langdon DW, Amato MP, Boringa J, Brochet B, Foley F, Fredrikson S, Hämäläinen P, Hartung HP, Krupp L, Penner IK, Reder AT, Benedict RH. Recommendations for a Brief International Cognitive Assessment for Multiple Sclerosis (BICAMS). Mult Scler. 2012;18(6):891-98.

[PMID:22190573]

http://dx.doi.org/10.1177/1352458511431076

17. Smith A. Symbol digit modalities test: Manual. Los Angeles (CA): Western Psychological Services; 1982.

18. Delis DC, Kramer JH, Kaplan E, Ober BA. California Verbal Learning Test, second edition (CVLT-II). San Antonio (TX): Psychological Corporation; 2000.

19. Benedict RH. The Brief Visuospatial Memory Test Revised (BVMT-R). Lutz (FL): Psychosocial Assessment Resources Inc; 1997.

20. Kurtzke JF. Rating neurologic impairment in multiple sclerosis: An expanded disability status scale (EDSS). Neurology. 1983;33(11):1444-52. [PMID:6685237]

http://dx.doi.org/10.1212/WNL.33.11.1444

21. Rousselet GA, Pernet CR. Improving standards in brainbehavior correlation analyses. Front Hum Neurosci. 2012; 6:119. [PMID:22563313]

http://dx.doi.org/10.3389/fnhum.2012.00119

22. Cohen J. Statistical power analysis for the behavioral sciences. 2nd ed. Hillsdale (NJ): Lawrence Erlbaum Associates; 1988.

23. WHO. Expert committee on physical status. Physical status: The use and interpretation of anthropometry. Geneva (Switzerland): World Health Organization; 1995.

24. Pilutti LA, Dlugonski D, Sandroff BM, Klaren RE, Motl RW. Internet-delivered lifestyle physical activity intervention improves body composition in multiple sclerosis: Preliminary evidence from a randomized controlled trial. Arch Phys Med Rehabil. 2014;95(7):1283-88.

[PMID:24699237] http://dx.doi.org/10.1016/j.apmr.2014.03.015

25. Parmenter BA, Testa SM, Schretlen DJ, Weinstock-Guttman $\mathrm{B}$, Benedict RH. The utility of regression-based norms in interpreting the minimal assessment of cognitive function in multiple sclerosis (MACFIMS). J Int Neuropsychol Soc. 2010;16(1):6-16. [PMID:19796441] http://dx.doi.org/10.1017/S1355617709990750

26. Kuo HK, Jones RN, Milberg WP, Tennstedt S, Talbot L, Morris JN, Lipsitz LA. Cognitive function in normalweight, overweight, and obese older adults: An analysis of the Advanced Cognitive Training for Independent and Vital Elderly cohort. J Am Geriatr Soc. 2006;54(1):97-103. 
JRRD, Volume 52, Number 3, 2015

\section{[PMID:16420204]}

http://dx.doi.org/10.1111/j.1532-5415.2005.00522.x

27. Sandroff BM, Motl RW. Fitness and cognitive processing speed in persons with multiple sclerosis: A cross-sectional investigation. J Clin Exp Neuropsychol. 2012;34(10): 1041-52. [PMID:22905722] http://dx.doi.org/10.1080/13803395.2012.715144

28. Benedict RH, Holtzer R, Motl RW, Foley FW, Kaur S, Hojnacki D, Weinstock-Guttman B. Upper and lower extremity motor function and cognitive impairment in multiple sclerosis. J Int Neuropsychol Soc. 2011;17(4):643-53. [PMID:21486517] http://dx.doi.org/10.1017/S1355617711000403

29. Dionyssiotis Y. Body composition in multiple sclerosis. Hippokratia. 2013;17(1):7-11. [PMID:23935336]

30. Motl RW, Pilutti LA, Sandroff BM. The importance of physical fitness in multiple sclerosis. J Nov Physiother. 2013;3(2):141-47. http://dx.doi.org/10.4172/2165-7025.1000141

31. Lambert CP, Lee Archer R, Evans WJ. Body composition in ambulatory women with multiple sclerosis. Arch Phys Med Rehabil. 2002;83(11):1559-61. [PMID:12422325] http://dx.doi.org/10.1053/apmr.2002.35663

32. Sioka C, Fotopoulos A, Georgiou A, Papakonstantinou S, Pelidou SH, Kyritsis AP, Kalef-Ezra JA. Body composition in ambulatory patients with multiple sclerosis. J Clin Densitom. 2011;14(4):465-70. [PMID:21835661] http://dx.doi.org/10.1016/j.jocd.2011.04.012
33. Benedict RH, Cookfair D, Gavett R, Gunther M, Munschauer F, Garg N, Weinstock-Guttman B. Validity of the minimal assessment of cognitive function in multiple sclerosis (MACFIMS). J Int Neuropsychol Soc. 2006;12(4): 549-58. [PMID:16981607] http://dx.doi.org/10.1017/S1355617706060723

34. Sandroff BM, Klaren RE, Pilutti LA, Dlugonski D, Benedict RH, Motl RW. Randomized controlled trial of physical activity, cognition, and walking in multiple sclerosis. J Neurol. 2014;261(2):363-72. [PMID:24323244] http://dx.doi.org/10.1007/s00415-013-7204-8

Submitted for publication September 4, 2014. Accepted in revised form February 11, 2015.

This article and any supplementary material should be cited as follows:

Sandroff BM, Hubbard EA, Pilutti LA, Motl RW. No association between body composition and cognition in ambulatory persons with multiple sclerosis: A brief report. J Rehabil Res Dev. 2015;52(3):301-8. http://dx.doi.org/10.1682/JRRD.2014.09.0208

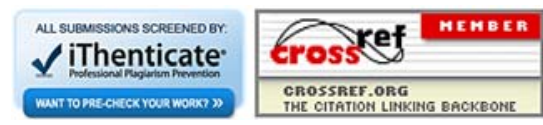

